\title{
Diagnostic Evidence of Recurrence/Relapse
}

National Cancer Institute

\section{Source}

National Cancer Institute. Diagnostic Evidence of Recurrence/Relapse. NCI Thesaurus.

Code C158779.

Evidence from testing or observation that there has been a recurrence or relapse. 